\title{
Modeling Symport/Antiport P Systems with a Class of Hierarchical Petri Nets ${ }^{\star}$
}

\author{
Luca Bernardinello, Nicola Bonzanni, Marco Mascheroni, and Lucia Pomello \\ Dipartimento di Informatica, Sistemistica e Comunicazione \\ Universit degli Studi di Milano-Bicocca \\ via Bicocca degli Arcimboldi 8, I-20126 Milano (Italy) \\ bernardinello@disco.unimib.it
}

Summary. A model of P systems with symport / antiport rules is given in terms of hypernets, a generalization of a class of hierarchical Petri nets introduced for modeling mobility inside the nets-within-nets paradigm. The hierarchical structure of a $\mathrm{P}$ system is reflected by the associated hypernet, where molecules are modeled by unstructured agents (simple tokens) and membranes by agents. Each agent is modeled by a net which may contain in its places unstructured agents or other agents. Agents can exchange tokens with their sub- or super-agents and thus the hierarchy may change. The main result of the paper shows a correspondence between reachable configurations of the $\mathrm{P}$ system and reachable hypermarkings of the related hypernet, in such a way that if the $\mathrm{P}$ system can evolve from one configuration to another one then in the hypernet there exists a corresponding transformation of hypermarkings.

\section{Introduction}

In recent years the notion of system of mobile agents has gained importance in computer science and engineering. These systems are formed by agents which move around a space, interacting with each other. Often, these agents are pieces of software traveling across a network of hosts, where they can be executed in a local environment. Such a development has led to envisage formal models in which one can represent mobile agents, their environment, and their interactions. Since agents move and run in parallel with others, concurrency theory is a natural framework in which to look for adequate models.

In 1986, Valk proposed a kind of Petri nets in which tokens can be nets, which can be moved across the places of a hosting net, possibly interacting with it (see [15]). Building on this idea, hypernets were defined in [1]. A hypernet is formed by agents, each modeled by a Petri net. In a given configuration, each agent, except one, is also a token residing in a place of another agent (the exception consists in the highest level agent, which acts as an environment for all others). The relation

\footnotetext{
* Partially supported by MIUR and CNR - IPI PAN.
} 
of containment can dynamically change as an effect of firing transitions; agents can exchange their sub-agents by forming so called consortia.

The hierarchy of agents in a hypernet resembles the hierarchy of membranes in a $\mathrm{P}$ system, and the mechanism of consortia can be seen as a way to exchange molecules across a membrane. This idea is the subject of the present paper, where we define a translation from $\mathrm{P}$ systems with symport/antiport rules to a class of hypernets. Such class is a generalization of the class defined in [1]. The main idea of this translation is quite simple: each membrane and each individual molecule in the $\mathrm{P}$ system is represented by an agent in the hypernet. Molecule agents are unstructured, that is, they are simple tokens, like in usual nets, and can only be passively moved by the active components. Membrane agents, viceversa, are nets, with places that can contain molecule agents, and places that can contain other membrane agents. Consortia correspond to rules of the P system, whereby molecules can be exchanged across a membrane.

It should be noted that hypernets would allow, in themselves, movement of membrane agents, so that the hierarchical structure of membranes could change. This capability is not exploited here, since we deal with $\mathrm{P}$ systems where only molecules move around, but might be useful in modeling more general kinds of systems.

In this paper, we are not interested in the computational aspects of the theory of $\mathrm{P}$ systems, but rather focus on modeling aspects. Consequently, we compare the two models on the basis of their reachable configurations.

After recalling the basic definitions related to the class of $\mathrm{P}$ systems with symport/antiport rules (Section 2), we define hypernets in Section 3. Section 4 shows how to build a hypernet from a $\mathrm{P}$ system, and states in which sense the two models can be considered as equivalent. Finally, in Section 5, we draw some considerations, and suggest possible developments.

\section{P Systems with Symport/Antiport Rules}

Many kinds of membrane systems have been investigated during the last years. One of the most studied variant of the general model of $\mathrm{P}$ systems was introduced in [10] under the name of systems with symport/antiport rules. Those terms came from two membrane transport mechanisms. Whereas the term symport stands for the biological process by which two molecules pass together across a membrane, when the two molecules pass simultaneously, but in the opposite direction, the process is called antiport.

The class of membrane systems with symport/antiport rules is a class of purely communicating $\mathrm{P}$ systems, where the objects involved in the computation only pass through membranes. This means that the objects involved never change and a sort of conservation law for objects is observed during the entire evolution of the system. 
Many results on this kind of $\mathrm{P}$ systems, especially about their computational power, can be found in [11],[7],[8],[4]. Here we provide a simplified version of the definition of $\mathrm{P}$ system with symport/antiport rules supplied by Păun [12].

\subsection{Formal definition}

Formally, we define a $\mathrm{P}$ system with symport/antiport rules (of degree $m$ ), as a construct of the form

$$
\Pi=\left(O, \mu, w_{1}, w_{2}, \ldots, w_{m}, R_{1}, R_{2}, \ldots, R_{m}\right)
$$

where:

- $O$ is the (finite and non empty) alphabet of objects

- the membrane structure $\mu=(N, E, i)$ is a rooted tree underlying $\Pi$, where $N=\{1,2, \ldots, m\}$ is the set of nodes and each node in $N$ defines a membrane of $\Pi$. The set $E \subseteq N \times N$ defines the edges. For each node $j \in N$, the membrane associated to the node $j$ contains all the membranes associated to the children of $j . i$ is the root of the tree and hence the skin membrane (the outermost membrane of the system)

- $w_{1}, w_{2}, \ldots, w_{m}$ are multisets over $O$ representing the objects present in the regions $1,2, \ldots, m$ of the membrane structure $\mu$ in the initial configuration of the system (in the following, multisets will be described either by strings, with exponents denoting the multiplicity of elements, or by the usual characteristic function of multisets) where the

- $R_{1}, R_{2}, \ldots, R_{m}$ are finite sets of evolution rules associated with the membranes of $\mu$. Moreover we impose $R_{i}=\emptyset$, where $i$ is the skin of the membrane structure. This clause ensures that the external membrane is impermeable and hence the total number of objects involved in the computation is finite (and constant); this is required if we want to build hypernets with a finite number of agents

In the following we often use the term molecule when referring to an object in a membrane of the $\mathrm{P}$ system.

As said above, each rule governs the communication through a specific membrane and can be of two kinds, symport rule or antiport rule. A symport rule is of the form $(u, i n)$ or $(u, o u t)$, where $u$ is a multiset over $O$, stating that all the objects of $u$ pass together through a membrane, entering in the former case and exiting in the latter. For example, in a membrane $i$, after the application of the symport rule $(u, i n)$, the multiset associated to this membrane will contain all the objects previously present, plus the objects present in $u$. The multiset associated to the membrane that contains $i$, will contain all the objects previously present, minus those in $u$. Similarly, an antiport rule is of the form $(u$, out; $v, i n)$, where $u$ and $v$ are multisets over $O$, stating that when $u$ exits, at the same time, a multiset $v$ must enter the membrane.

The $\mathrm{P}$ system described above evolves from configuration to configuration by the application of a multiset of rules in each membrane. Formally, a configuration 
is a tuple $C=\left(v_{1}, v_{2}, \ldots, v_{m}\right)$ and $C \stackrel{\hat{R}}{\Rightarrow} C^{\prime}$ denotes that $C$ evolves into $C^{\prime}$ due to the application of $\hat{R}$, where $\hat{R}=\left(\bar{R}_{1}, \bar{R}_{2}, \ldots, \bar{R}_{m}\right)$ is a multi-rules vector applicable to $C$ and $\bar{R}_{j}$ is a multiset over $R_{j}$.

The evolution of the system is non-deterministic and maximally parallel: at each step, the configuration changes by applying a maximal multiset of rules, chosen in a non deterministic way; the rules must be all applicable without mutual interferences in the current configuration.

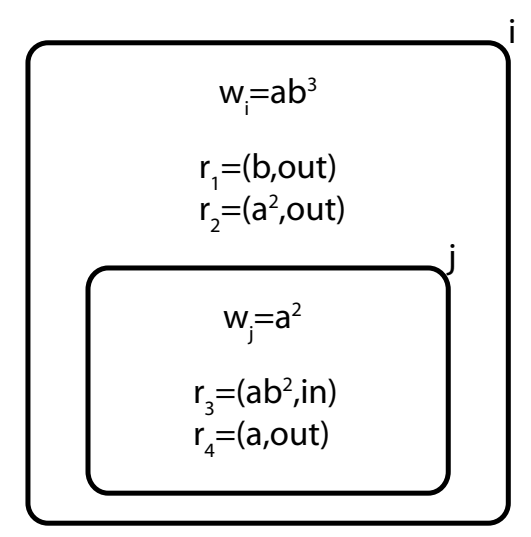

Fig. 1. Fragment of a symport/antiport $\mathrm{P}$ system

\subsection{Example}

Fig. 1 shows a fragment of a $\mathrm{P}$ system with symport rules. The system depicted here consists of two nested membranes: $j$, the inner membrane, and $i$, the outer one, which we assume to be a membrane contained in a larger membrane structure. The set of rules of $i$ is $R_{i}=\left\{(b, o u t),\left(a^{2}, o u t\right)\right\}$, and the set of rules of $j$ is $R_{j}=\left\{\left(a b^{2}, i n\right),(a, o u t)\right\}$. In the same way we define the initial multisets of objects $w_{i}=a b^{3}$ and $w_{j}=a^{2}$.

In this configuration the rules $r_{1}, r_{3}, r_{4}$ are enabled and a multi-rules vector can be built with this rules in a maximally parallel manner, i.e.: the multi-rules vector $\hat{R}=\left(\left\{r_{1}\right\},\left\{r_{3}, r_{4}, r_{4}\right\}\right)$ is applicable to the initial configuration. Note that other multi-rules vectors can be applied to the same configuration. The application of $\hat{R}$ leads to a new state where the objects in the membrane $i$ are $a^{2}$ and the objects in the membrane $j$ are $a b^{2}$.

\section{Hypernets}

In this section we introduce a generalization of Petri hypernets [1] that for simplicity we call also here hypernets. A hypernet is defined by a fixed set of agents, each 
agent is modeled by a net and can manipulate other agents as tokens, while being manipulated as token by another agent at the same time. This yields a hierarchy of agents. The highest level agent acts as an environment for all other agents, these latter are located each one in some place of another agent. Agents can exchange tokens with their sub- or super-agents and thus the hierarchy may change.

In what follows we first define the structure of hypernets giving the definition of agent and of hypernet, then we define the behavior of hypernets, and at the end of the section we illustrate hypernets on an example modeling the $\mathrm{P}$ system given in Subsection 2.2 as it will be discussed in Section 4 .

\subsection{Structure of hypernets}

An agent is modeled by a Petri net, a bipartite oriented graph, whose nodes are of two types: places and transitions. Places are partitioned into two disjoint sets: the set of local places, which are locations in which other agents can stay, and the set of virtual places, which represent communication channels along which agents exchange tokens each others. Places and transitions are interconnected by weighted oriented arcs, which define how many tokens are taken away from an input place and how many are put into an output place, when a transition fires. For each transition the sum of the weights of the input arcs must be equal to the sum of the weights of the output arcs. In this way the amount of tokens will not change while transitions fire. Moreover, to each triple of interconnected elements place-transition-place it is assigned, by a function $\phi_{A}$, a value which defines, in a way compatible with the arc weights, the number of tokens which flow along the path identified by the triple. In other words, $\phi_{A}$ defines how the tokens taken away from an input place of a transition will be distributed into the output places, when the transition will fire; and this distribution will be the same for each occurrence of the same transition. For basic definitions and notions on Petri nets, see, for example, [14].

Definition 1. An agent is a tuple $A=\left(P_{A} \cup V_{A}, T_{A}, F_{A}, \phi_{A}\right)$, where $\left(P_{A} \cup\right.$ $\left.V_{A}, T_{A}, F_{A}\right)$ is a, possibly empty, finite Petri net in which :

- $P_{A}$ is the set of local places and $V_{A}$ is the set of virtual places, (or communication places), with $P_{A} \cap V_{A}=\emptyset$;

- $T_{A}$ is the set of transitions;

- the function $F_{A}:\left(\left(V_{A} \cup P_{A}\right) \times T_{A}\right) \cup\left(T_{A} \times\left(V_{A} \cup P_{A}\right)\right) \longrightarrow \mathbb{N}$ defines the flow, assigning a weight to each arc identified by the pair of elements $x, y$ such that: $F_{A}(x, y)>0$, in such a way that $: \forall t \in T_{A}, \sum_{p \in \bullet} F_{A}(p, t)=\sum_{p \in t} \bullet F_{A}(t, p)$, where $p \in{ }^{\bullet} t$ iff $F_{A}(p, t)>0$ and $p \in t^{\bullet}$ iff $F_{A}(t, p)>0$;

and the function $\phi_{A}:\left(V_{A} \cup P_{A}\right) \times T_{A} \times\left(V_{A} \cup P_{A}\right) \longrightarrow \mathbb{N}$ defines the paths, i.e.: the triples $(p, t, q)$ such that: $\phi_{A}(p, t, q)>0$, by assigning a weight to them in such a way that:

$$
\begin{aligned}
& \forall p \in \bullet t, F_{A}(p, t)=\sum_{q \in t} \bullet \phi_{A}(p, t, q) \\
& \forall q \in t^{\bullet}, F_{A}(t, q)=\sum_{p \in \bullet} \phi_{A}(p, t, q)
\end{aligned}
$$


In the following $(p, t, q) \in \phi_{A}$ iff $\phi_{A}(p, t, q)>0$, moreover, given a subset of agents $X \subseteq \mathcal{N}$, we use the following notation: $P_{X}=\bigcup_{A \in X} P_{A}, V_{X}=\bigcup_{A \in X} V_{A}$, $T_{X}=\bigcup_{A \in X} T_{A}, \phi_{X}=\bigcup_{A \in X} \phi_{A}$.

A hypernet is defined by a set of agents and by a relation $\Delta$. Agents have disjoint sets of places. A transition may belong to different agents, modeling synchronous interaction among them. Transitions connected to virtual places model interchanges of tokens among sub-/super-agents. Said output paths the path ending with a virtual place and input paths the ones starting with a virtual place, the relation $\Delta$ identifies communication channels by defining, for a given transition belonging to different agents, a correspondence (output path - input path) in a way compatible with path weights.

Definition 2. Let $\mathcal{N}=\left\{A_{1}, A_{2}, \ldots, A_{n}\right\}$ be a family of agents, and let $S^{o}=$ $\left\{(p, t, v) \in \phi_{A} \mid A \in \mathcal{N}\right.$ and $\left.v \in V_{A}\right\}$ and $S^{i}=\left\{(v, t, q) \in \phi_{A} \mid A \in \mathcal{N}\right.$ and $\left.v \in V_{A}\right\}$ be the sets of output paths and input paths, respectively. (Note that a path can be both an output and an input path.)

$A$ hypernet is a pair $H=(\mathcal{N}, \Delta)$, where

- The agents in $\mathcal{N}$ have disjoint sets of places:

$$
\forall A_{i}, A_{j} \in \mathcal{N},\left(P_{A_{i}} \cup V_{A_{i}}\right) \cap\left(P_{A_{j}} \cup V_{A_{j}}\right)=\emptyset ;
$$

- and $\Delta \subseteq S^{o} \times S^{i}$ is a relation which associates, for a given transition, output paths to input paths with the same weight and belonging to different agents, i.e.:

$\forall t \in T_{\mathcal{N}}, \forall(p, t, q) \in \phi_{A_{i}}$ and $\forall\left(p^{\prime}, t, q^{\prime}\right) \in \phi_{A_{j}}$ such that $A_{i}, A_{j} \in \mathcal{N}:$

$\left((p, t, q),\left(p^{\prime}, t, q^{\prime}\right)\right) \in \Delta \Rightarrow A_{i} \neq A_{j}$ and $\phi_{A_{i}}(p, t, q)=\phi_{A_{j}}\left(p^{\prime}, t, q^{\prime}\right)$.

Definition 3. Let $\mathcal{N}=\left\{A_{1}, A_{2}, \ldots, A_{n}\right\}$ be a family of agents. A map $\mathcal{M}$ : $\left\{A_{2}, \ldots, A_{n}\right\} \longrightarrow P_{\mathcal{N}}$, assigning to each agent different from $A_{1}$ the local place in which is located, is a hypermarking of $\mathcal{N}$ iff, considering the relation $\uparrow_{\mathcal{M}} \subseteq \mathcal{N} \times \mathcal{N}$ defined by : $A_{i} \uparrow_{\mathcal{M}} A_{j} \Leftrightarrow \mathcal{M}\left(A_{i}\right) \in P_{A_{j}}$, then the graph $\left\langle\mathcal{N}, \uparrow_{\mathcal{M}}\right\rangle$ is a tree with $\operatorname{root} A_{1}$.

Definition 4. A marked hypernet is a pair $(H, \mathcal{M})$ where $H$ is a hypernet and $\mathcal{M}$ is a hypermarking defining the initial configuration.

In a configuration the system results hierarchically structured. The highest level agent $A_{1}$, the root of the tree describing the hierarchy, plays the role of the environment containing all the other agents. The relation of containment between agents, and then the hierarchical structure, can change as an effect of firing transitions as formalized in the following subsection.

\subsection{Behaviour of hypernets}

Let $H=(\mathcal{N}, \Delta)$, with $\mathcal{N}=\left\{A_{1}, A_{2}, \ldots, A_{n}\right\}$, be a hypernet.

A consortium is a set of interconnected active agents, cooperating in performing a transition $t$, moving other passive agents along the paths containing $t$. 
Definition 5. A consortium is a tuple $\Gamma=(t, \tau, \delta, \gamma)$ where:

- $t \in T_{\mathcal{N}}$ is the name of the consortium,

- $\tau \subseteq\left\{A \in \mathcal{N} \mid t \in T_{A}\right\}, \tau \neq \emptyset$, is the non empty set of active agents. To this set we can associate $\phi_{\tau_{t}}=\left\{(p, t, q) \in \phi_{\tau} \mid p, q \in P_{\tau} \cup V_{\tau}\right\}$, the set of paths of the agents $\tau$ containing the transition $t$.

- $\delta$ defines a bijective correspondence between output paths containing $t$ and input paths containing $t$ of active agents, without contradicting the relation $\Delta$. Let $\phi_{o, \tau_{t}}=\phi_{\tau_{t}} \cap S^{o}$ and $\phi_{i, \tau_{t}}=\phi_{\tau_{t}} \cap S^{i}$. If $\phi_{o, \tau_{t}} \neq \emptyset, \delta: \phi_{o, \tau_{t}} \longrightarrow \phi_{i, \tau_{t}}$ is a bijection such that: $\forall s \in \phi_{o, \tau_{t}}, \delta(s)=s^{\prime} \Rightarrow\left(s, s^{\prime}\right) \in \Delta$, while if $\phi_{o, \tau_{t}}=\emptyset$, then $\delta$ is the empty map. Note that $\delta$ relates paths belonging to different agents.

- The passive agents which are moved when the consortium occurs are selected through the map $\gamma$. Let $C \subseteq \mathcal{N} \backslash A_{1}$ be a chosen set of passive agents, then $\gamma: C \longrightarrow \phi_{\tau_{t}} \backslash S^{i}$ is surjective and associates as many passive agents to each path containing $t$ and belonging to an active agent as the weight of the path itself, i.e.: $\forall s \in \phi_{\tau_{t}} \backslash S^{i},\left|\gamma^{-1}(s)\right|=\phi_{\mathcal{N}}(s)$. Note that an agent can be active and passive at the same time.

Moreover the following conditions must be satisfied:

- the set of active agents $\tau$ is a minimal one, in the sense that the agents in $\tau$ must be each other interconnected through the interaction $t$, i.e.:

the undirected graph $G_{1}=\left(\tau, E_{1}\right)$ is connected,

where $E_{1}=\left\{\left(A_{i}, A_{j}\right) \mid A_{i}, A_{j} \in \tau\right.$ and $\left.\exists s_{i} \in \phi_{A_{i}}, \exists s_{j} \in \phi_{A_{j}}: \delta\left(s_{i}\right)=s_{j}\right\}$ and

- the undirected graph $G_{2}=\left(\tau \cup C, E_{2}\right)$ is acyclic,

where $E_{2}$ connects $A_{i}$ to $A_{j}$ if $A_{i}$ will be put inside $A_{j}$ throught, i.e.: considered the recursively defined map $\delta^{*}: \phi_{\tau_{t}} \longrightarrow \phi_{\tau_{t}}$ such that

$$
\begin{gathered}
\delta^{*}(s)= \begin{cases}s & \text { if } s \notin \phi_{o, \tau_{t}} \\
\delta^{*}(\delta(s)) & \text { otherwise }\end{cases} \\
E_{2}=\left\{\left(A_{i}, A_{j}\right) \mid \delta^{*}\left(\gamma\left(A_{i}\right)\right) \in \phi_{A j}, A_{i} \in C, A_{j} \in \tau\right\} .
\end{gathered}
$$

The intuition behind the last condition of the previous definition is the following. By subsequent applications of the map $\delta$ it is possible to construct chains of paths interrelated through paths with only virtual places. However, the meaningful chains are the one which starts with a path with a real input place, the one from which an agent will be taken out, and ends with a path with a real output place, the one in which the agent will be put into. The last condition requires that these chains are not closed.

In [2] it is proven that chains containing a real place can be prolonged to finite chains containing at most two real places, one in an input path and one in an output path. 
Definition 6. Let $H=(\mathcal{N}, \Delta)$ be a generalized hypernet and $\mathcal{M}$ be a hypermarking.

A consortium $\Gamma=(t, \tau, \delta, \gamma)$ is enabled in $\mathcal{M}$, denoted $\mathcal{M}[\Gamma\rangle$, iff the following two conditions hold

- $\forall A \in C, \gamma(A)=(p, t, q) \Rightarrow \mathcal{M}(A)=p$

- $\forall A_{i}, A_{j} \in \tau, \forall s \in S^{o} \cap \phi_{A_{i}}, \delta(s) \in \phi_{A_{j}} \Rightarrow A_{i} \uparrow_{\mathcal{M}} A_{j} \vee A_{j} \uparrow_{\mathcal{M}} A_{i}$

If $\mathcal{M}[\Gamma\rangle$, then the occurrence of $\Gamma$ leads to the new hypermarking $\mathcal{M}^{\prime}$, denoted $\mathcal{M}[\Gamma\rangle \mathcal{M}^{\prime}$, such that $\forall A \in \mathcal{N}$ :

$$
\mathcal{M}^{\prime}(A)= \begin{cases}\mathcal{M}(A) & \text { if } A \notin C ; \\ q & \text { if } A \in C \text { and } \delta^{*}(\gamma(A))=(p, t, q) .\end{cases}
$$

It is possible to prove [2] that $\mathcal{M}^{\prime}$ is a hypermarking, i.e.: that the class of hypermarkings of a hypernet is closed under the occurrence of a consortium.

Two consortia $\Gamma_{1}=\left(t_{1}, \tau_{1}, \delta_{1}, \gamma_{1}\right)$ and $\Gamma_{2}=\left(t_{2}, \tau_{2}, \delta_{2}, \gamma_{2}\right)$ are independent iff the maps $\gamma_{1}$ and $\gamma_{2}$ select two different sets of passive agents, i.e.: iff $C_{1} \cap C_{2}=\emptyset$

If two independent consortia are both enabled in a hypermarking $\mathcal{M}$ then they can concurrently occur in $\mathcal{M}$.

Let $\Gamma_{H}$ be the set of possible consortia in $H$. A set of consortia $U \subseteq \Gamma_{H}$ is a step enabled in a hypermarking $\mathcal{M}$, denoted $\mathcal{M}[U\rangle$, iff

- $\forall \Gamma_{i}, \Gamma_{j} \in U, \Gamma_{i}$ and $\Gamma_{j}$ are independent,

- $\forall \Gamma_{i} \in U, \mathcal{M}\left[\Gamma_{i}\right\rangle$

If $\mathcal{M}[U\rangle$, then the occurrence of the step $U$ leads to the new hypermarking $\mathcal{M}^{\prime}$, denoted $\mathcal{M}[U\rangle \mathcal{M}^{\prime}$, such that $\forall A \in \mathcal{N}$ :

$$
\mathcal{M}^{\prime}(A)= \begin{cases}\mathcal{M}(A) & \text { if } \forall \Gamma_{i} \in U, A \notin C_{i} ; \\ q & \text { if } \exists \Gamma_{i} \in U:\left(A \in C_{i} \text { and } \delta_{i}^{*}\left(\gamma_{i}(A)\right)=\left(p, t_{i}, q\right)\right) .\end{cases}
$$

$U$ is a maximal step enabled in $\mathcal{M}$, and its occurrence yields $\mathcal{M}^{\prime}$, iff $\mathcal{M}[U\rangle \mathcal{M}^{\prime}$ and $\forall U^{\prime} \supset U: \operatorname{not}\left(\mathcal{M}\left[U^{\prime}\right\rangle\right)$.

In [2] it is shown how it is possible to associate to each hypernet a 1-safe net in such a way that there is a strict correspondence between their behaviors, i.e., in terms of Petri net theory, in such a way that the case graph of the 1-safe net is isomorphic to the transition system generated by the reachable hypermarkings of the hypernet.

Since 1-safe nets are a basic class model in Petri net theory, this translation shows that hypernets are well rooted inside the theory of Petri nets.

\subsection{Example}

The Fig. 2 shows the structure of two hypernet's agents. The unfilled circles are local places while the filled ones are virtual places. The agent $A_{j}$ is nested in the 


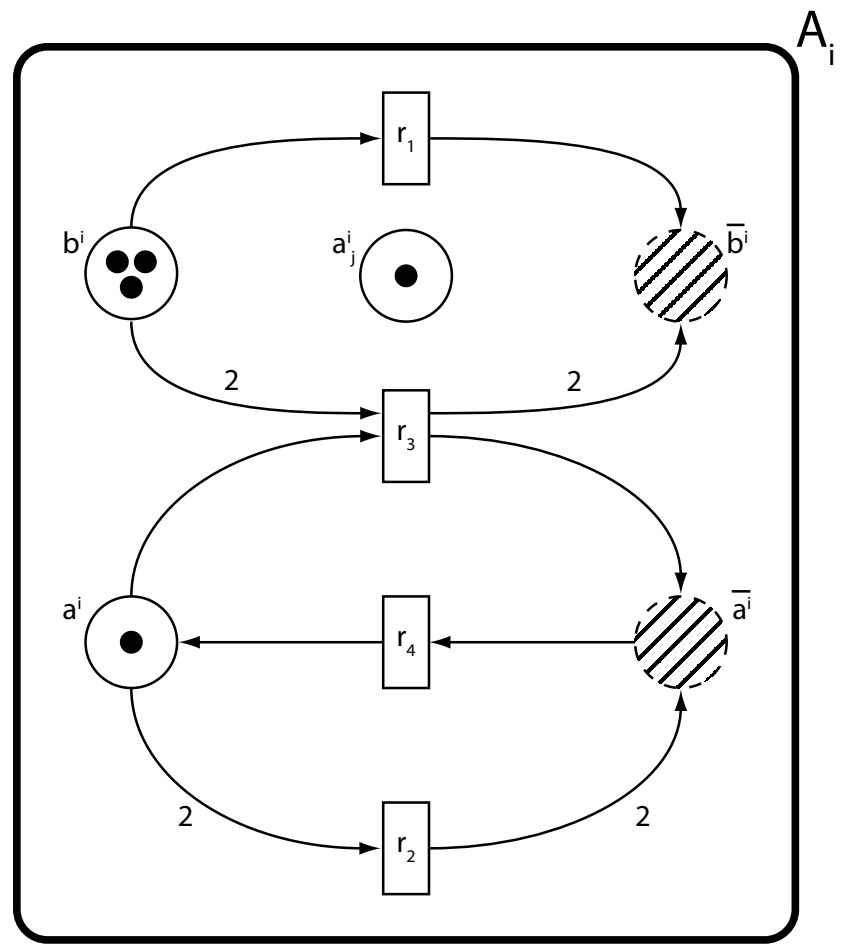

(a)

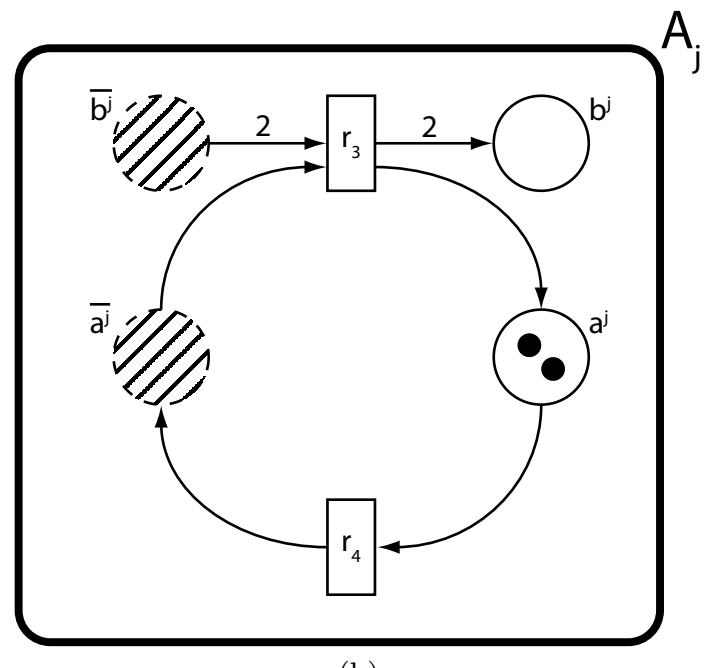

(b)

Fig. 2. Fragment of a hypernet 
agent $A_{i}$, in fact $\mathcal{M}\left(A_{j}\right)=a_{j}^{i}$, so $A_{j} \uparrow_{\mathcal{M}} A_{i}$. Moreover we assume $u_{1}, u_{2}, u_{3}, u_{4}$ to be unstructured agents such that $\mathcal{M}\left(u_{1}\right)=\mathcal{M}\left(u_{2}\right)=\mathcal{M}\left(u_{3}\right)=b^{i}$ and $\mathcal{M}\left(u_{4}\right)=a^{i}$. Now consider the consortium $\Gamma=\left(r_{3}, \tau, \delta, \gamma\right)$ where

- the set of active agents is $\tau=\left\{A_{i}, A_{j}\right\}$,

- the bijection $\delta$ builds two communication channels between $A_{i}$ and $A_{j}$ gluing two pair of paths: $\delta\left(a^{i}, r_{3}, \bar{a}^{i}\right)=\left(\bar{a}^{j}, r_{3}, a^{j}\right)$ and $\delta\left(b^{i}, r_{3}, \bar{b}^{i}\right)=\left(\bar{b}^{j}, r_{3}, b^{j}\right)$,

- the set of passive agents is $C=\left\{u_{1}, u_{2}, u_{4}\right\}$ and $\gamma\left(u_{1}\right)=\gamma\left(u_{2}\right)=\left(b^{i}, r_{3}, \bar{b}^{i}\right)$ and $\gamma\left(u_{4}\right)=\left(a^{i}, r_{3}, \bar{a}^{i}\right)$.

The consortium $\Gamma$ is valid and enabled in the initial hypermarking. When $\Gamma$ occurs the system reaches a new hypermarking $\mathcal{M}^{\prime}$ where $\mathcal{M}^{\prime}\left(u_{1}\right)=\mathcal{M}^{\prime}\left(u_{2}\right)=b^{j}$ and $\mathcal{M}^{\prime}\left(u_{4}\right)=a^{j}$. Note that the agents $u_{1}, u_{2}, u_{4}$ pass through the communication channels established by $\delta$ from the agent $A_{i}$ to the agent $A_{j}$.

\section{Membrane Systems as Hypernets}

Our goal in this section is to show how a $\mathrm{P}$ system with symport/antiport rules and with an impermeable external membrane can be modeled as a hypernet.

In the following, we write $i \triangleleft j$ to mean that membrane $i$ is directly contained in membrane $j$.

Let $\Pi=\left(O, \mu, w_{1}, \ldots, w_{m}, R_{1}, \ldots, R_{m}\right)$ be a $\mathrm{P}$ system of degree $m$, with symport/antiport rules. We assume that 1 is the outer membrane, with no rules, so that $R_{1}=\emptyset$.

The hypernet associated to $\Pi$ will be denoted by $H=(\mathcal{N}, \Delta)$. The hypernet $H$ contains one agent for each membrane, and one agent for each individual molecule in $\Pi$. Notice that, in the P-systems we handle, molecules are neither created nor deleted.

Let $W=\sum_{i=1}^{m} w_{i} . W$ is a multiset giving the total number of objects for each type in the system. Define

$$
\mathrm{MOL}=\{(x, i) \mid x \in O \wedge 1 \leq i \leq W(x)\}
$$

For each $(x, i)$ in MOL, we define an unstructured agent in the hypernet $H$.

$$
\mathcal{N}=\left\{A_{1}, A_{2}, \ldots, A_{m}\right\} \cup \mathrm{MOL}
$$

Agent $A_{i}$ corresponds to membrane $i$ of the $\mathrm{P}$ system. It has one place for each membrane directly contained in $i$, and one for each type of molecule; moreover, it has one virtual place for each type of molecule, to be used in exchanging tokens.

$$
\begin{gathered}
P_{i}=\left\{a_{j}^{i} \mid j \triangleleft i\right\} \cup\left\{x^{i} \mid x \in O\right\} \\
V_{i}=\left\{\bar{x}^{i} \mid x \in O\right\}
\end{gathered}
$$

The set of transitions of agent $A_{i}$ has one transition for each rule in membrane $i$, and one for each rule in membranes directly contained in $i$. 


$$
T_{i}=\left\{r \mid r \in R_{i}\right\} \cup\left\{r \mid r \in R_{j} \wedge j \triangleleft i\right\}
$$

We now turn to define the flow function and the paths for agent $A_{i}$.

- For each rule $r=(u, i n) \in R_{i}$, and for each rule $r=(u, i n ; v$, out $) \in R_{i}$ :

$$
F\left(\bar{x}^{i}, r\right)=F\left(r, x^{i}\right)=\phi\left(\left(\bar{x}^{i}, r, x^{i}\right)\right)=u(x)
$$

- For each rule $r=(v$, out $) \in R_{i}$, and for each rule $r=(u, i n ; v, o u t) \in R_{i}$ :

$$
F\left(x^{i}, r\right)=F\left(r, \bar{x}^{i}\right)=\phi_{i}\left(\left(x^{i}, r, \bar{x}^{i}\right)\right)=v(x)
$$

Let $j \triangleleft i$. Then,

- For each rule $r=(u, i n) \in R_{j}$, and for each rule $r=(u, i n ; v$, out $) \in R_{j}$ :

$$
F\left(x^{i}, r\right)=F\left(r, \bar{x}^{i}\right)=\phi_{i}\left(\left(x_{i}, r, \bar{x}^{i}\right)\right)=u(x)
$$

- For each rule $r=(v$, out $) \in R_{j}$, and for each rule $r=(u, i n ; v$, out $) \in R_{j}$ :

$$
F\left(\bar{x}^{i}, r\right)=F\left(r, x_{i}\right)=\phi_{i}\left(\left(\bar{x}^{i}, r, x_{i}\right)\right)=v(x)
$$

Define now the $\Delta$ relation. For all $i, j$ such that $i \triangleleft j$ :

$$
\begin{array}{r}
\forall r=(u, \text { in }) \in R_{i}, \forall x \in O: u(x)>0,\left(x^{j}, r, \bar{x}^{j}\right) \Delta\left(\bar{x}^{i}, r, x^{i}\right) \\
\forall r=(u, \text { out }) \in R_{i}, \forall x \in O: u(x)>0,\left(x^{i}, r, \bar{x}^{i}\right) \Delta\left(\bar{x}^{j}, r, x^{j}\right) \\
\forall r=(u, \text { in } ; v, \text { out }) \in R_{i}, \forall x \in O: u(x)>0,\left(x^{j}, r, \bar{x}^{j}\right) \Delta\left(\bar{x}^{i}, r, x^{i}\right) \\
\forall r=(u, \text { in } ; v, \text { out }) \in R_{i}, \forall x \in O: v(x)>0,\left(x^{j}, r, \bar{x}^{j}\right) \Delta\left(\bar{x}^{i}, r, x^{i}\right)
\end{array}
$$

The initial hypermarking $\mathcal{M}$ reflects the initial configuration of $\Pi$. Membrane agents are placed according to the hierarchical structure of $\Pi$ :

$$
\forall i \in\{2, \ldots, m\}: \mathcal{M}\left(A_{i}\right)=a_{i}^{j} \text { iff } i \triangleleft j
$$

All agents $(x, k)$ corresponding to molecules are initially distributed in the corresponding places $x^{i}$ in membrane agents so that a place $x^{i}$ contains $w_{i}(x)$ unstructured agents of type $(x, k)$.

In order to state the exact relation between the dynamics of a $\mathrm{P}$ system $\Pi$ and the dynamics of the corresponding hypernet $H$, we need to define two relations. The first defines a correspondence between configurations of $\Pi$ and hypermarkings of $H$. The other defines a correspondence between steps of $\Pi$ and maximal steps of $H$. Define

$$
\text { Conf }=\left\{\left(v_{1}, \ldots, v_{m}\right) \mid \sum_{1}^{m} v_{i}=\sum_{1}^{m} w_{i}\right\}
$$

as the set of all potential configurations of $\Pi$ with the same number and type of molecules as the initial configuration. Define $\mathbf{H M}$ as the set of all hypermarkings of $H$. 
Let $\mathcal{M}:\left\{A_{1}, \ldots, A_{m}\right\} \cup \mathrm{MOL} \rightarrow P$ be an element of $\mathbf{H M}$, where $P$ is the set of all local places of $H$, and $C=\left(v_{1}, \ldots v_{m}\right) \in$ Conf, where $v_{i}: O \rightarrow \mathbb{N}$. We also need some auxiliary definition. By $I(x, i, \mathcal{M})$ we denote the set of agents representing molecules of type $x$ hosted in the corresponding place of agent $A_{i}$ in $\mathcal{M}$.

$$
I(x, i, \mathcal{M})=\left\{(x, i) \mid(x, i) \in \mathrm{MOL} \wedge \mathcal{M}((x, i))=x^{i}\right\}
$$

Definition 7. The hypermarking $\mathcal{M}$ simulates configuration $C$ (denoted by $\mathcal{M} \sim$ C) iff

1. $\mathcal{M}\left(A_{i}\right) \in P_{j}$ iff $i \triangleleft j$, for $i \in\{2, \ldots, m\}$

2. $|I(x, i, \mathcal{M})|=v_{i}(x)$

Notice that $\sim$ is a partial surjective function: each configuration of $\Pi$ has at least one corresponding hypermarking. The hypermarkings corresponding to one given configuration differ only for the distribution of molecules of the same kind in membrane agents. These molecules are identical in the $\mathrm{P}$ system, while their corresponding agents are distinguished.

We now define a correspondence between maximal steps in the $\mathrm{P}$ system and maximal steps of consortia in the hypernet. This correspondence is based on another one, associating single rules and consortia.

Let $r$ be a rule of membrane $i$ in $\Pi$. By construction, the associated hypernet has two transitions labeled by $r$, one in the agent corresponding to $i$, and one in the agent corresponding to the membrane containing $i$; assume it is $j$. A consortium simulating the execution of $r$ involves $i$ and $j$ as active agents, and a number of passive agents taken from MOL.

We consider here an antiport rule $r=(u, i n ; v, o u t)$, where $u$ and $v$ are multiset on $O$. Symport rules can be seen as special cases where either $u$ or $v$ is the empty multiset.

Definition 8. Let $\Gamma=(r, \tau, \delta, \gamma)$ be a consortium. Then $\Gamma \sim r$ iff the following conditions hold.

1. $\tau=\left\{A_{i}, A_{j}\right\}$

2. The output paths involved in $\Gamma$ are either of the form $\left(y^{i}, r, \bar{y}^{i}\right)$ if $v(y)>0$, or of the form $\left(x^{j}, r, \bar{x}^{j}\right)$ if $u(x)>0$.

3. The function $\delta$ is defined by

$$
\begin{gathered}
\delta\left(\left(y^{i}, r, \bar{y}^{i}\right)\right)=\left(\bar{y}^{j}, r, y^{j}\right) \\
\delta\left(\left(x^{j}, r, \bar{x}^{j}\right)\right)=\left(\bar{x}^{i}, r, x^{i}\right)
\end{gathered}
$$

4. Let $Z=\left\{z \in \mathrm{MOL} \mid z=(x, k) \wedge \gamma(z)=\left(x^{j}, r, \bar{x}^{j}\right)\right\}$; then $|Z|=u(x)$

5. Let $Z=\left\{z \in \mathrm{MOL} \mid z=(y, k) \wedge \gamma(z)=\left(y^{i}, r, \bar{y}^{i}\right)\right\} ;$ then $|Z|=v(y)$

A transition in a $\mathrm{P}$ system is a multiset of independently executable rules. Let $R=\bigcup_{i=1}^{m} R_{i}$ be the set of all rules of $\Pi$, and $\rho: R \rightarrow \mathbb{N}$ be a multiset of rules. A set $U$ of consortia in $H$ simulates $\rho$ (denoted by $U \sim \rho$ ) if, for each $r \in R$, 
$U$ contains $\rho(r)$ consortia which simulate $r$, and the consortia in $U$ are pairwise independent.

We are now ready to state the main result of this section. The following lemma states that any change of configuration in $\Pi$ can be simulated by a set of mutually independent consortia in $H$. Let $\Pi$ be a P-system with symport and antiport rules, such that 1 is the outer membrane with $R_{1}=\emptyset$, and $H=(\mathcal{N}, \Delta)$ be the associated hypernet, with initial hypermarking $\mathcal{M}$.

Lemma 1. Let $C$ be a configuration of $\Pi$, and $\rho$ be a multiset of rules, enabled at $C$, with $C \stackrel{\rho}{\Rightarrow} C^{\prime}$. Then, for all $\mathcal{M} \in \mathbf{H M}$,

$$
\mathcal{M} \sim C \Rightarrow \exists U \subseteq \Gamma_{H}: U \sim \rho, \mathcal{M}[U\rangle \mathcal{M}^{\prime}, \mathcal{M}^{\prime} \sim C^{\prime}
$$

Notice that the consortia forming $U$ can always be chosen to be pairwise independent. From this lemma, one can prove, by induction from the initial configuration, that the evolution of the $\mathrm{P}$ system can be simulated by the hypernet.

\subsection{Example}

Fig. 2, already discussed above (Section 3) as a generic hypernet, shows the fragment of the hypernet corresponding to the P system of Fig. 1. The two membranes $i$ and $j$ are modeled by the agents $A_{i}$ (Fig. 2(a)) and $A_{j}$ (Fig. 2(b)). The local place $a_{j}^{i} \in P_{i}$, which contains (as token) the agent $A_{j}$, reflects the fact that the membrane $j$ is nested inside $i$, while the local places $a^{i}, b^{i}$ represent the presence of molecules $a$ and $b$ respectively, inside the agent $A_{i}$ (this is also true for $a^{j}, b^{j}$ and the membrane $j$ ). Then $\left\{r_{1}, r_{2}, r_{3}, r_{4}\right\} \subseteq T_{\mathcal{N}}$ are transitions built from the evolution rules of the membrane system. The initial hypermarking matches the initial configuration of the $\mathrm{P}$ system.

\section{Conclusions}

In this paper we have considered $\mathrm{P}$ systems with symport/antiport rules and we have shown how they can be modeled by a class of hierarchical Petri net systems, a generalization of hypernets [1].

The hierarchical structure of the P system is reflected by the agents's hierarchy of the hypernet, where molecules are modeled by unstructured agents (hence empty nets or simple tokens) and membranes by agents, nets which may contain in their places unstructured agents or other agents.

The exchange of molecules through a membrane of a $\mathrm{P}$ system, as defined by a symport or an antiport rule, corresponds to a consortium involving two active agents, that represent the two nested membranes which exchange each other passive unstructured tokens (molecules).

The main result, as given in Section 4, states a correspondence between reachable configurations of the $\mathrm{P}$ system and reachable hypermarkings of the related 
hypernet. If the $\mathrm{P}$ system can evolves from a configuration to another one as the result of the application of a multi-rules vector, then in the hypernet exists an associated set of consortia transforming a hypermarking, corresponding to the first configuration, and another corresponding to the second one.

A translation, that takes a hypernet and returns a 1-safe Petri net (one of the basic models in Petri net theory) such that the case graphs of the latter is isomorphic to the transition system generated by the execution of consortia of the former, has been shown in [2]. This transformation proves that hypernets are well rooted in net theory. In [9] a definition of non sequential processes for hypernets was given. This can be used to derive an alternative semantics for $\mathrm{P}$ systems based on a purely causal dependency notion.

In the literature other works have investigated the relation between $\mathrm{P}$ system and Petri nets [6], [13], [5]. It is a matter of future work a deeper comparison with these approaches and with other computational models, inspired by biological membranes and derived from calculi of concurrency and mobility, as for example those proposed by Cardelli [3].

Hypernets allow movement of structured agents from one level to another one, so that the hierarchy of agents may change. In terms of $\mathrm{P}$ systems, this means to consider movements of membrane agents. This capability is not exploited here, however it would be interesting in future to study the modeling of $\mathrm{P}$ systems with active membranes [12].

Acknowledgements. The authors wish to thank Claudio Zandron and Alberto Leporati for their helpful suggestions.

\section{References}

1. M.A. Bednarczyk, L. Bernardinello, W. Pawłowski, L. Pomello: Modelling mobility with Petri Hypernets, LNCS 3423, Springer, 2005, 28-44.

2. N. Bonzanni: P systems e reti di Petri ad alto livello, Universit degli studi di MilanoBicocca. Dipartimento di Informatica Sistemistica e Comunicazione. Graduation thesis, 2007.

3. L. Cardelli: Brane Calculi. In Computational Methods in Systems Biology, LNCS 3082, Springer, 2005, 257-278.

4. P. Frisco: About P systems with symport/antiport, Soft Computing, 9, 9 (2005), 664-672.

5. P. Frisco: P Systems, Petri Nets, and Program Machines, LNCS 3850, Springer, 2006, 209-223.

6. J.H.C.M. Kleijn, M. Koutny, G. Rozenberg: Towards a Petri Net Semantics for Membrane Systems, LNCS 3850, Springer, 2006, 292-309.

7. C. Martín-Vide, A. Păun, Gh. Păun: On the power of P systems with symport rules, J. of Universal Computer Science, 8, 2 (2002), 317-331.

8. C. Martín-Vide, A. Păun, Gh. Păun, G. Rozenberg: Membrane systems with coupled transport: Universality and normal forms, Fundamenta Informaticae, 49, 1-3 (2002), $1-15$. 
9. M. Mascheroni: Hypernet e processi non sequenziali, Universit degli studi di MilanoBicocca. Dipartimento di Informatica Sistemistica e Comunicazione. Graduation thesis, 2007.

10. A. Păun, Gh. Păun: The Power of Communication: P systems with Symport/Antiport, New Generation Computing, 20, 3 (2002), 295-305.

11. A. Păun, Gh. Păun, G. Rozenberg: Computing by Communication in networks of Membranes, Intern. J. of Foundations of Computer Sci., 13, 6 (2002), 779-798.

12. Gh. Păun: Introduction to Membrane Computing, First brainstorming Workshop on Uncertainty in Membrane Computing, Palma de Mallorca, Spain, 2004.

13. Z. Qi, J. You, H. Mao: P Systems and Petri Nets, LNCS 2933, Springer, 2004, 286303.

14. W. Reising, G. Rozenberg, eds.: Lectures on Petri Nets I: Basic Models, LNCS 1491, Springer, 1998.

15. R. Valk: Nets in Computer Organisation. In Advances in Petri Nets (W. Brauer, W. Reising, G. Rozenberg, eds.), LNCS 255, Springer, 1986, 218-233. 
